\title{
Single giant mediastinal rhabdomyoma as a sole manifestation of TSC in foetus
}

\author{
Marek Godavaa, , Hana Filipovac, Lubomir Dubravad, Radek Vrtel', Kamila Michalkova ${ }^{\mathrm{e}}$, Maria Janikovaf, \\ Lenka Bakaj-Zbrozkovae, Jiri Navratilg
}

\begin{abstract}
Background. Presence of multiple cardiac rhabdomyomas is one of the major features of Tuberous sclerosis (TSC), but isolated progressing single giant rhabdomyoma is very rare and not typical of TSC.

Case report. This report presents family without obvious history of TSC with occurrence of giant mediastinal rhabdomyoma affecting the haemodynamics in male foetus, without other TSC symptoms. Girl from the next gravidity had prenatally detected multiple rhabdomyomas and small subcortical tuber of brain detected after birth. DNA analysis found novel c.4861 A > T TSC2 variant and large deletion in TSC2 in tumour tissue from male foetus. The novel TSC2 variant was also present in the girl and her healthy father, in silico analysis suggested its functional effect on TSC2. Brain MRI of the father detected mild TSC specific abnormality.

Conclusion. We suggest the novel TSC2 mutation is a cause of mild TSC in this family and has reduced expression. The clinical and molecular findings in this family also emphasize that TSC diagnosis should be also evaluated in case of single giant foetal cardiac rhabdomyoma.
\end{abstract}

Key words: cardiac rhabdomyoma, TSC2, reduced expression, mild tuberous sclerosis.

Received: February 19, 2017; Accepted: May 4, 2017; Available online: June 12, 2017

https://doi.org/10.5507/bp.2017.023

${ }^{a}$ Centre of Fetal Medicine and Medical Genetics, FETMED, Olomouc, Czech Republic

'Laboratory of Medical Genetics, SPADIA LAB a.S., Novy Jicin, Czech Republic

'Department of Medical Genetics, University Hospital Olomouc, Czech Republic

${ }^{d}$ Centre of Prenatal Diagnosis, U.S.G. POL s.r.o., Prostejov, Czech Republic

'Department of Radiology, University Hospital Olomouc, Czech Republic

${ }^{f}$ Department of Clinical and Molecular Pathology, Faculty of Medicine and Dentistry, Palacky University Olomouc, Czech Republic

${ }^{g}$ Outpatient Cardiology Department, Pediatric Clinic, The University Hospital Brno, Czech Republic

Corresponding author: Marek Godava, e-mail: GodavaM@seznam.cz

\section{INTRODUCTION}

The rhabdomyomas are the most common type of cardiac tumours in foetuses, newborns and children ${ }^{1}$. Presence of multiple cardiac rhabdomyomas is one of the major features of tuberous sclerosis (TSC) and they can often be detected in the prenatal period. In contrast, single rhabdomyoma is not typical of TSC and giant mediastinal / cardiac tumour is a very rare finding in TSC $\left(\right.$ ref. $\left.^{2,3}\right)$. Here we report on a family without history of TSC with a huge mediastinal tumour in one foetus, multiple cardiac rhabdomyomas of foetus in the next gravidity and a novel TSC2 variant found in 3 members of the family.

\section{CASE REPORT}

The foetus from the second gravidity showed increased nuchal translucence (NT) in the first trimester but the karyotype was $46, \mathrm{XY}$. During the USG follow up, there was a progression in NT thickness and echocardiography performed in $17+5$ g.h. revealed the presence of a probable rhabdomyoma measuring $5 \times 6 \mathrm{~mm}$ close to the mitral valve and tendency to bradycardia. According to the USG evaluation in the cardiologic centre, the finding was not typical of rhabdomyomatosis in the 21st week of pregnancy. There was presence of pathological structures heading from the area of dorsal mediastinum and around heart atria, infiltrating the atrial walls and growing into the atrium. The mass was filling approximately one half of the right atrium cavity. A dense large structure (measuring $10 \times 13 \times 14 \mathrm{~mm}$ ) was present in the pericardium with pericardial effusion. Due to the poor prognosis for the foetus, the parents opted for termination of the pregnancy in the 22nd week of gravidity. Autopsy described a male foetus without signs of external malformation. Chest organs were in common positions but there was a bulging mass under the pericardium. The tumour measured $16 \mathrm{x}$ $12 \times 8 \mathrm{~mm}$, was on the surface of the left atrium and partly on the left ventricle surface and was tightly connected with the myocardium. A small amount of clear pleural fluid was present. Histology of the tumour confirmed presence of cardiac rhabdomyoma. Other organs were without signs of malformation.

In the next gravidity, slight hyperechogenicity of the mitral valve and posterior wall of the left heart ventricle was detected in the 26th week of pregnancy. Subsequent USG follow-up detected intracardial structures resembling rhabdomyomas in the left atrium and left ventricle in $31+2$ g.h. The rhabdomyomas did not impair heart haemodynamic and had a non-progressive character. 
Table 1. Result of in silico analysis of novel TSC2 mutation functional effect.

\begin{tabular}{ll}
\hline Online predictor & Result of in silico analysis \\
\hline SIFT, PROVEAN & damaging, deleterious (for all of the assessed protein isoforms) \\
PolyPhen2 & probably damaging, MSA*: Ile 1621 is conserved in mammals \\
Mutationassessor & medium functional impact \\
Mutation Taster & disease causing, PhyloP score: 3.362 \\
\hline
\end{tabular}

* MSA - multiple sequence alignment

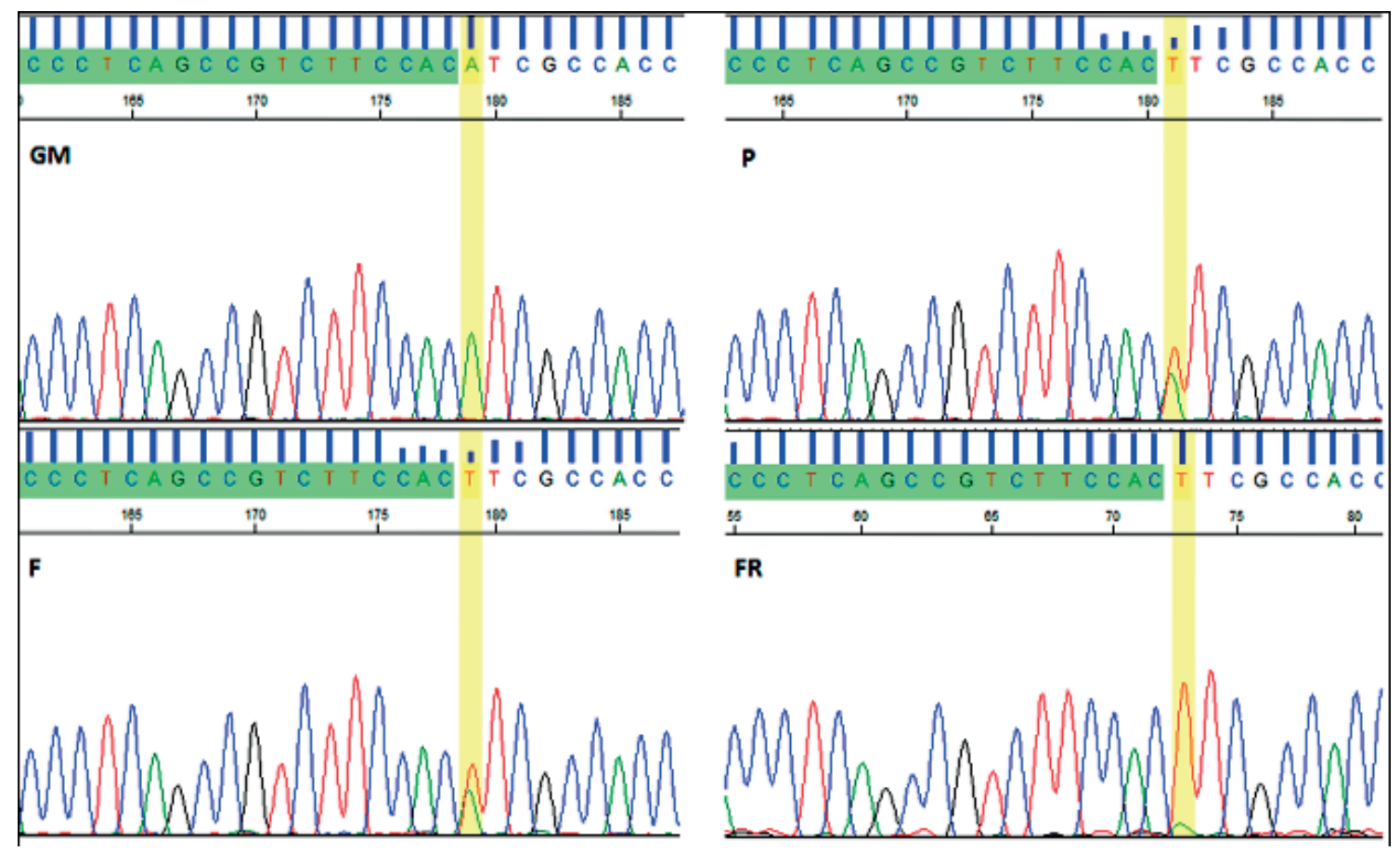

Fig. 1. The sequenograms demonstrate heterozygous presence of c.4861A>T TSC2 mutation (NM_000548.4, ENST00000219476) in father (F) and proband (P), and wild type sequence in paternal grandmother (GM).

There is homozygous constitution of the mutation in DNA isolated from cardiac rhabdomyoma of foetus (FR) from second gravidity of proband's mother - the mutation actually affects only one allele, the second one is affected by deletion (according to the MLPA analysis).

Spontaneous delivery occurred in the 39th week of pregnancy; the girl had no obvious respiratory or cardiac troubles. Neonatal echocardiography described multiple rhabdomyomas without haemodynamic significance.

The family was referred to the genetic department when the girl was 6 weeks old; she showed no obvious TSC symptoms, psychomotor development was normal. Brain MRI examination at 9 weeks of life showed the presence of a tiny hypersignal lesion measuring $2.5 \mathrm{~mm}$ in the borderline of subcortical and centrum ovale regions of left frontal lobe - presumed to be a cortical/subcortical tuber. During the MRI follow up (at 1 year and 5 months and at 2 years and 6 months) the left frontal tuber showed no expansive character and another thin stripped lesion was uncovered just next to the body of the right lateral ventricle. The parents and older sister had no obvious TSC specific skin lesions or dental pits.

\section{Results of genetic analysis}

The Sanger sequencing of all coding regions in TSC1 and TSC2 was done with the informed consent of the parents - the unknown c.4861A>T / p.Ile1621Phe muta- tion in 38th exon of TSC2 (Fig. 1) was found in the proband. The mutation was present in the father, but absent in older sister, mother and grandmothers. In the next step, we assessed the effect of the mutation on TSC 2 protein using available online predictors. All of the five in silico tools predicted the TSC 2 mutation has a functional impact (Table 1). And more, the mutant allele was neither found in ExAC nor The thousand genomes databases. The absence of the variant in the databases also supports the functional impact of the novel TSC 2 mutation.

DNA analysis found the presence of the novel TSC2 mutation in foetus (Fig. 1). The TSC2 is a tumour suppressor and therefore, should the novel TSC 2 mutation have functional impact, then the cardiac tumour should contain second (somatic) mutation. Tumour and nontumour tissues in foetal heart tissue (FFPE sample) were separated by a microdissection instrument and DNA was isolated. MLPA analysis of foetal cardiac rhabdomyoma tissue found the presence of exons 3-42 deletion in TSC2 that was absent in normal myocardial tissue (Fig. 2). This finding also supported the functional impact of the novel TSC2 mutation. 


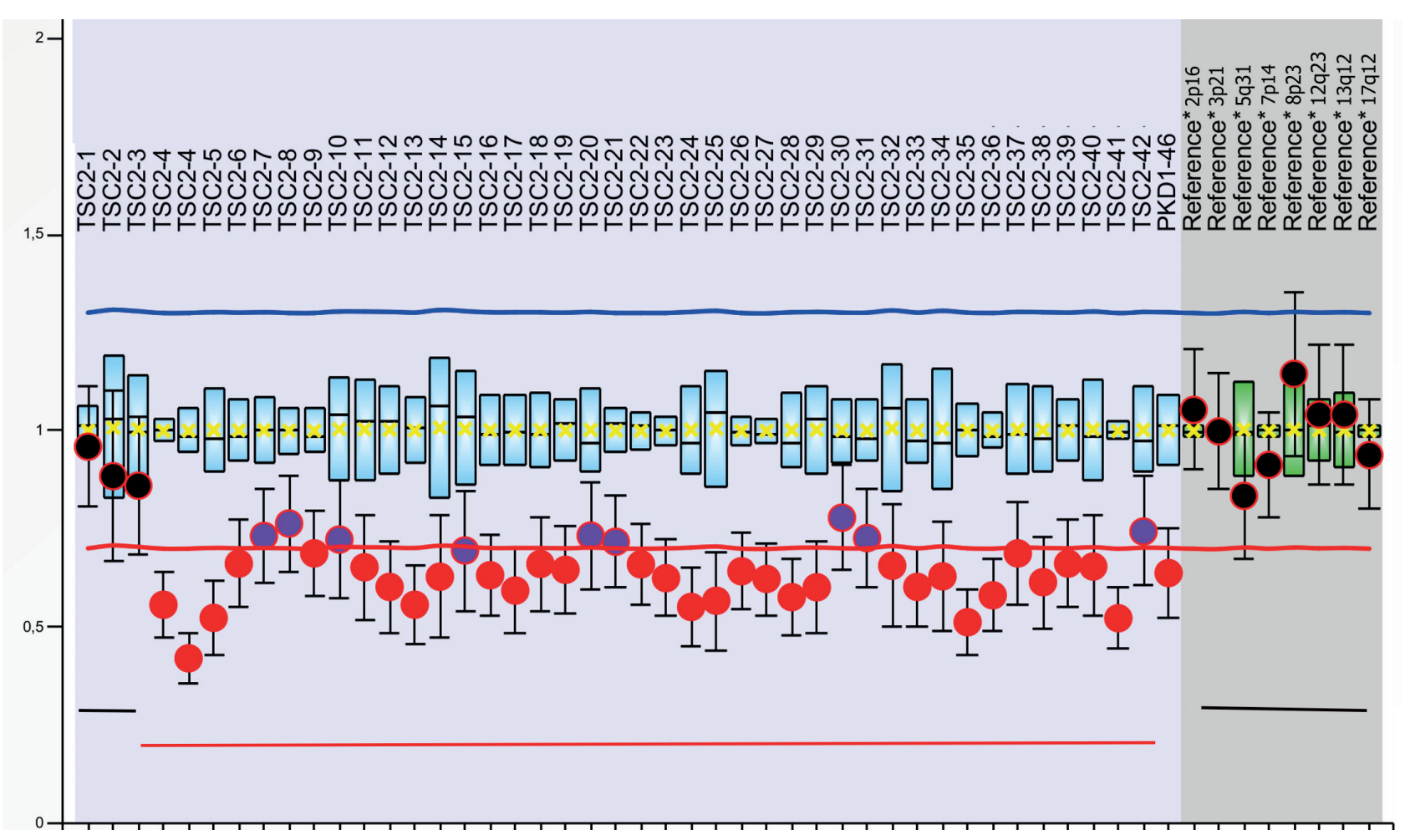

Fig. 2. The ratio chart displays results of MLPA analysis of TSC2 (P046-C1 TSC2, Lot C1-0315, MRC Holland), analysis was performed by Coffalyser software. Analysed DNA was isolated from cardiac rhabdomyoma tissue (foetus from second pregnancy of proband's mother). In the case of clear deletion, signal of probe (red dots) lies below the red line. There is a deletion of exons 4-42 of TSC2 and at least exon 46 of PKD1. Partially reduced signal of some probes (the exons 7, 8, 10, 15, 20, 21, 30, 31 and 42 of TSC2, purple dots) represent heterogeneity of cardiac tissue - tumour cells could not be entirely separated from cells of normal heart tissue. This finding demonstrates presence of the second hit (loss of heterozygosity) in TSC2 in foetal cardiac rhabdomyoma. The red straight line in the bottom of the picture represents schematic extent of TSC2/PKD1 deletion.

TSC shows variable expressivity but the penetrance should be complete, therefore we searched for other signs of TSC in the father. Although his abdominal USG scan did not find any pathology of the kidney, the brain MRI revealed the presence of fine hyperintensive streaks, suggestive of subcortical tubers, in the frontotemporal region. In $\mathrm{T} 2$ sequences, there were tiny hyperintesive nodes (not larger than $2 \mathrm{~mm}$ ) in the caudothalamic region. The supratentorial changes described on brain MRI were therefore consistent with diagnosis of TSC.

\section{DISCUSSION}

The p.Ile1621Phe mutation is located in GAP domain of TSC2 that is crucial for inactivating Rheb (Rheb activates mTORC1 complex). Several causal mutations were described in this region. Hoogeveen-Westerveld et al. ${ }^{4}$ performed functional assessment of several TSC2 variants, including variants surrounding the p.Ile1621Phe mutation. They proved that p.His1620Arg and p.Thr1623Ile mutations were less effective at inhibiting mTORC1, these mutations showed significantly higher mean T389/S6K ratio than wild-type TSC2. The ratio was not different from causal p.Arg611Gln mutation.

The proband and her father also have rs 13335638 polymorphism (intron 37 ). Neither this polymorphism nor the c.4861A>T TSC 2 mutation was detected in the paternal grandmother. This indicates the mutation should originate from the paternal grandfather - he had renal disease from his twenties, underwent two surgeries of the kidney and died of end stage renal disease at the age of 42. Unfortunately, his medical records are not available; therefore possible TSC symptoms related to kidney disease could not be excluded. The mutation could, however, be of de novo origin in the father and the kidney problems in the paternal grandfather may have been caused by an unrelated process. Interestingly, the paternal origin of TSC2 mutation in a family with very mild symptoms of TSC was also assumed in a report by Mayer et al. ${ }^{5}$.

TSC shows variable severity of symptoms, there is both interfamilial and intrafamilial variability of phenotype expression. Women usually tend to have milder disease than men.

The sporadic TSC2 mutations are linked with more severe phenotype of TSC as has been observed in cases without a family history of TSC. In cases of familial TSC2 mutations, the phenotype is less severe ${ }^{6}$ and there are some reports of mildly affected TSC families also presenting with prenatally detected multiple cardiac rhabdomyomas $^{5,7}$. Multiple foetal / neonatal cardiac rhabdomyomas were significantly associated with TSC; they can be found in up to $95 \%$ cases of TSC (ref. ${ }^{8,9}$ ). In contrast, the occurrence of single intracardiac rhabdomyoma is not overrepresented in persons with TSC compared to non TSC patients $^{2}$ and single giant progressing rhabdomyoma is 
very rare in persons with TSC. Schlaegel et al. ${ }^{10}$ described a case similar to our family - a foetus showing a large mediastinal tumour with pleural effusion and mediastinal shift at 27 weeks of gravidity and negative TSC focused family history. The tumour increased in size during the USG follow up. A second smaller intracardiac tumour appeared at 36 weeks of pregnancy. The cardiac findings were confirmed in the postnatal period, rhabdomyomas were without haemodynamic compromise. The CNS pathology was uncovered only postnatally, the neurological examination of neonate had normal findings. Genetic analysis confirmed TSC. During the follow up USG pathology of kidney was not detected and the cardiac tumours showed spontaneous regression (MRI examination showed the presence of only one rhabdomyoma measuring $33 \mathrm{~mm}$ ).

Some large cardiac rhabdomyomas could require surgical intervention (because of haemodynamic compromise), but oral administration of everolimus (mTOR inhibitor) may be also considered ${ }^{11}$. CNS pathology may be detected prenatally; therefore brain MRI examination of foetuses with cardiac rhabdomyomas is recommended ${ }^{12}$. The mTOR inhibitors are now used to treat a variety of TSCassociated symptoms. Moreover, there is also evidence of successful treatment of brain pathologies by rapamycine in the prenatal period in well established mouse TSC models ${ }^{13,14}$. Although these results allow for the potential of prenatal mTOR inhibitors administration to treat large cardiac rhabdomyomas that are seriously affecting haemodynamic, more research is needed prior to the treatment of human foetuses with TSC - mice treated prenatally with rapamycine showed some level of cognitive function impairment $^{13}$.

\section{CONCLUSION}

Cardiac rhabdomyomas tend to regress/diminish in a high proportion of TSC cases within the first few years of life. This is also the case for our proband. She is now almost 4 years old and her cardiac rhabdomyomas are regressing. Although rare, the cardiac rhabdomyoma could appear again, therefore regular follow up by a cardiologist is reasonable for some time (at least until the onset of puberty) for all TSC patients ${ }^{15}$. With reference to the family described in our report, we suggest that TSC should be also considered in any case of single giant mediastinal rhabdomyoma regardless of TSC focused family history.

Acknowledgement: We would like to thank Dr. Petr Zapletal (Dept. of Pathology, Prostejov Hospital) for providing us with the FFPE sample of foetal heart sample.

Author contributions: MG, HF, RV: study design; $\mathrm{HF}$ : DNA analysis; MJ, HF: foetal tissue analysis; MG, HF: data interpretation; LD, JN, KM, LB-Z: cardiologic ex- amination and neuroimaging; $\mathrm{MG}, \mathrm{HF}$ : paper preparation (equally contribution).

Conflict of interest statement: The authors state that there are no conflicts of interest regarding the publication of this article. This work received no grant support.

\section{REFERENCES}

1. Carrilho MC, Tonni G, Araujo Júnior E. Fetal cardiac tumours: prenatal diagnosis and outcomes. Rev Bras Cir Cardiovasc 2015;30(1):VI-VII.

2. Verhaaren HA, Vanakker O, De Wolf D, Suys B, François K, Matthys D. Left ventricular outflow obstruction in rhabdomyoma of infancy: meta-analysis of the literature. J Pediatr 2003;143(2):258-63.

3. Lee KA, Won HS, Shim JY, Lee PR, Kim A. Molecular genetic, cardiac and neurodevelopmental findings in cases of prenatally diagnosed rhabdomyoma associated with tuberous sclerosis complex. Ultrasound Obstet Gynecol 2013;41:306-11.

4. Hoogeveen-Westerveld M, Ekong R, Povey S, Mayer K, Lannoy N, Elmslie F, Bebin M, Dies K, Thompson C, Sparagana SP, Davies P, van Eeghen AM, Thiele EA, van den Ouweland A, Halley D, Nellist M. Functional assessment of TSC2 variants identified in individuals with tuberous sclerosis complex. Hum Mutat 2013;34:167-75.

5. Mayer K, Goedbloed M, van Zijl K, Nellist M, Rott HD. Characterisation of a novel TSC2 missense mutation in the GAP related domain associated with minimal clinical manifestations of tuberous sclerosis. J Med Genet 2004;41:e64.

6. Sancak O, Nellist M, Goedbloed M, Elfferich P, Wouters C, MaatKievit A, Zonnenberg B, Verhoef S, Halley D, van den Ouweland A. Mutational analysis of the TSC1 and TSC2 genes in a diagnostic setting: genotype-phenotype correlations and comparison of diagnostic DNA techniques in Tuberous Sclerosis Complex. Eur J Hum Genet 2005; 13:731-41.

7. Chen CP, Su YN, Hung CC, Shih JC, Wang W. Novel mutation in the TSC2 gene associated with prenatally diagnosed cardiac rhabdomyomas and cerebral tuberous sclerosis. J Formos Med Assoc 2006;105:599-603.

8. Chao AS, Chao A, Wang TH, Chang YC, Chang YL, Hsieh CC, Lien R, Su WJ. Outcome of antenatally diagnosed cardiac rhabdomyoma: case series and a meta-analysis. Ultrasound Obstet Gynecol 2008;31:28995.

9. Tworetzky W, McElhinney DB, Margossian R, Moon-Grady AJ, Sallee D, Goldmuntz E, van der Velde ME, Silverman NH, Allan LD. Association between cardiac tumours and tuberous sclerosis in the foetus and neonate. Am J Cardiol 2003;92:487-9.

10. Schlaegel F, Takacs Z, Solomayer EF, Abdul-Kaliq, H., MeybergSolomayer, G. Prenatal diagnosis of giant cardiac rhabdomyoma with fetal hydrops in tuberous sclerosis. J Prenat Med 2013;7:39-41.

11. Mlczoch E, Hanslik A, Luckner D, Kitzmüller E, Prayer D, MichelBehnke I. Prenatal diagnosis of giant cardiac rhabdomyoma in tuberous sclerosis complex: a new therapeutic option with everolimus. Ultrasound Obstet Gynecol 2015;45(5):618-21.

12. Mühler MR, Rake A, Schwabe M, Schmidt S, Kivelitz D, Chaoui R, Hamm B. Value of fetal cerebral MRI in sonographically proven cardiac rhabdomyoma. Pediatr Radiol 2007;37:467-74.

13. Way SW, Rozas NS, Wu HC, McKenna J 3rd, Reith RM, Hashmi SS, Dash PK, Gambello MJ. The differential effects of prenatal and/or postnatal rapamycin on neurodevelopmental defects and cognition in a neuroglial mouse model of tuberous sclerosis complex. Hum Mol Genet 2012;21(14):3226-36.

14. Tsai PT, Greene-Colozzi E, Goto J, Anderl S, Kwiatkowski DJ, Sahin M. Prenatal rapamycin results in early and late behavioral abnormalities in wildtype C57BL/6 mice. Behav Genet 2013;43(1):51-9.

15. Thatte NM, Guleserian KJ, Veeram Reddy SR. New-onset cardiac rhabdomyoma beyond infancy in a patient with tuberous sclerosis complex. Cardiol Young 2016;26:396-9. 